STROKE

Intelligent, mobile stroke imaging

A machine learning algorithm can accurately and quickly identify large vessel occlusions (LVOs) from CT angiograms obtained in mobile stroke units, investigators have reported in Stroke. The approach could reduce time to treatment and improve patient outcomes.

Patients with LVOs can often be treated with endovascular thrombectomy, and earlier treatment improves outcomes. The ability to identify patients with LVOs in the pre-hospital setting would facilitate earlier treatment. Mobile stroke units provide an opportunity for such pre-hospital triage, as they often include facilities for CT angiography (CTA), which detects LVOs. Machine learning algorithms for automated analysis of CTA images acquired in mobile stroke units could further improve pre-hospital triage.

Sunil Sheth and colleagues had previously developed a machine learning algorithm known as DeepSymNet for analysis of CTA images acquired in hospital. However, CTA images acquired in mobile stroke units can be of lower and variable quality. In their new study, Sheth and colleagues investigated the performance of an updated machine learning algorithm, called DeepSymNet-v2, with CTA images acquired in mobile stroke units.

The researchers trained DeepSymNet-v2 with 870 CTA images acquired in hospital, and tested its accuracy with 441 further in-hospital images. They then applied the algorithm to CTA images acquired from 68 individuals in two mobile stroke units in the USA - one in Houston and one in Los Angeles.

DeepSymNet-v2 identified LVOs with high accuracy - the area under the receiver operating characteristic curve was 0.80 . This accuracy was achieved with an algorithm running time $<1 \mathrm{~min}$. The investigators conclude that their algorithm could reduce the amount of time lost in pre-hospital triage and accelerate the transfer of patients with LVOs to thrombectomy-capable centres for earlier treatment.

lan Fyfe

ORIGINAL ARTICLE Czap, A. L. et al. Machine learning automated detection of large vessel occlusion from mobile stroke unit computed tomography angiography. Stroke https://doi.org/10.1161/STROKEAHA.121.036091 (2021) RELATED ARTICLE Bivard, A. et al. Artificial intelligence for decision support in acute stroke - current roles and potential. Nat. Rev. Neurol. 16, 575-585 (2020)

\title{
Safety and efficacy of COVID-19 vaccines in people with neurological disorders
}

In people with neurological disorders, COVID-19 vaccine uptake is likely to be influenced by concerns over adverse effects and loss of efficacy related to the disease and/or its treatment. Emerging evidence, as exemplified by two recent publications, should help to provide some reassurance regarding the safety and efficacy of COVID-19 vaccination in this patient population.

COVID-19 vaccines are administered by intramuscular injection, and in individuals with neuromuscular disease, loss of muscle mass, combined with steroid treatment, could limit the immune response to the vaccine. However, a new study involving 14 patients with neuromuscular disorders shows that these individuals mounted a comparable antibody response to that observed in healthy control participants. Moreover, vaccination produced higher antibody levels than did SARS-CoV-2 infection in patients with these conditions.

The second study examined COVID-19 vaccine uptake and outcomes in 491 people with epilepsy, compared with 217 people with neuropsychiatric conditions and 273 healthy volunteers. The epilepsy group showed lower levels of vaccine uptake and higher levels of vaccine hesitancy than the other two groups. A small proportion (less than 10\%) of patients with epilepsy who underwent vaccination reported an increase in seizure frequency afterwards. In some cases, however, this effect might have been attributable to withdrawal or reduction of antiseizure medication owing to fears over drug interactions with the vaccine.

Although both teams acknowledge the need for more research, their studies provide some encouraging indications regarding the safety and efficacy of COVID-19 vaccines in people with neurological disorders.

Heather Wood

ORIGINAL ARTICLES Demonbreun, A. R. et al. mRNA intramuscular vaccination produces a robust lgG antibody response in advanced neuromuscular disease. Neuromuscul. Disord. https://doi.org/10.1016/j.nmd.2021. 11.006 (2021) | Lu, L. et al. COVID- 19 vaccine take- up rate and safety in adults with epilepsy: data from a multicenter study in China. Epilepsia https://doi.org/10.1111/epi.17138 (2021)

\section{DEMENTIA}

\section{Role for endogenous retrovirus in FTD}

Levels of human endogenous retrovirus $\mathrm{K}$ (HERV-K) are elevated in the blood of individuals with behavioural variant frontotemporal dementia (bvFTD), according to a new study published in Communications Medicine. The findings highlight a novel aspect of bvFTD pathology and suggest that HERV-K is a potential biomarker for the disease.

HERV-K belongs to a family of viruses whose genetic code was incorporated into the human genome during evolution. Previous studies found that serum levels of HERV-K are elevated in individuals with amyotrophic lateral sclerosis (ALS) and are associated with TAR DNA-binding protein 43 (TDP43) pathology. Many individuals with bvFTD have TDP43 pathology, so the aim of the new study was to establish whether HERV-K levels are also elevated in these individuals.

The researchers measured levels of HERV-K DNA in the serum of 63 people with bvFTD, 89 people with ALS and 21 control participants. HERV-K DNA levels were higher in participants with bvFTD and ALS than in control participants.
To establish whether HERV-K could be used as a biomarker for bvFTD, the researchers generated receiver operating characteristic curves. HERV-K could be used to distinguish participants with bvFTD from control participants with high accuracy - the area under the curve was 0.867 .

The researchers also measured the levels of mRNA for HERV-K in post-mortem samples. In a brain region known to be affected by TDP43 pathology, HERV-K mRNA levels were higher in individuals with bvFTD than in control participants. This finding was not replicated in the cerebellum, which is largely unaffected by FTD pathology, suggesting a link between HERV-K and TDP43 pathology in bvFTD.

These findings indicate that HERV-K expression is perturbed in bvFTD, opening up new avenues of research into the pathogenesis of the disease and providing a potential new biomarker.

Sarah Lemprière

ORIGINAL ARTICLE Phan, K. et al. Pathological manifestation of human endogenous retrovirus $\mathrm{K}$ in frontotemporal dementia. Commun. Med. 1, 60 (2021) 